\title{
A Framework for Segmentation of Inhomogeneous Live Cell Images using Fractional Derivatives and Level Set Method
}

\author{
Sarabpreet Kaur \\ Department of Electrical Engineering \\ Indian Institute of Technology \\ Ropar
}

\author{
J.S. Sahambi \\ Department of Electrical Engineering \\ Indian Institute of Technology \\ Ropar
}

\begin{abstract}
Cell segmentation has gained significant importance in modern biological image processing applications. The commonly used image segmentation algorithms are region based and depend on the homogeneity of the intensities of the pixels in the region of interest. But due to the highly inhomogeneous behavior of cell nuclei and background, feature overlapping between the two regions lead to misclassification and poor segmentation results. This paper proposes a method to segment the cell images taking into consideration the intensity inhomogeneity issue. A fractional differential term has been introduced in the clustering criteria for bias correction for improving the homogeneity of the cell images. A method to optimize the fractional order for images has also been proposed. Further an improved narrow band level set method using Chan Vese model has been proposed to improve the computational speed of the algorithm. The proposed method is evaluated on datasets of $2 \mathrm{D}$ microscopy images and images with improved homogeneity have been obtained. The results also show improved segmentation results and the time efficient bahaviour of the proposed method.
\end{abstract}

\section{Keywords}

Intensity inhomogeneity, Fractional derivatives, Level sets, Chan Vese mode

\section{INTRODUCTION}

Most of the cell studies depend on the analysis of large cell clusters with the help of microscopy imaging techniques. [13][12]. For studying the intracellular phenomena, fluorescence microscopy is often used as it allows biologists to perform live cell experiments with high sensitivity and specificity. Analysis of cell populations give information about average cell characteristic and the dynamic behavior of the cells. The complexity of performing the cell analysis is increasing with large volume of the image data being analyzed. Manual processing of such data is time consuming and error prone, creating a demand for automated techniques [17][11]. Thus, automated analysis of cellular structure has become a need to describe complex biological processes.

Cell segmentation is one of the most basic processing steps in many biological cell image applications. Automatic segmentation is much more time efficient compared to time consuming manual processes. However, automated cell segmentation techniques are much more challenging. These challenges arise from different image acquisition techniques and complex topology of the cells such as touching cells and overlapping cells [4]. Some of the image acquisition techniques require the images to be captured in a controlled environment in order to take care of the biological phenomenon resulting in poor image quality such as in phase contrast images. In the case of fluorescent images, low concentration of fluorescent label needs to be applied to limit the effect of cytotoxicity on the cells. This results in a low signal to noise ratio in the image data, making the task of automated detection more challenging. The topological changes include shape deformation, close contact and overlap of cells. Different stages of cell cycle such as mitosis and apoptosis add to the complexity of the problem. The segmentation algorithms proposed in the recent past produced efficient results on regions with little or no cell crowding, however they could not separate touching cells accurately. The watershed algorithm is one of the most commonly used segmentation method to overcome this challenge. However it often results in over segmentation [20]. Intensity inhomogeneity has been a big issue in cell segmentation. Segmentation is considerably difficult for inhomogeneous images due to overlapping between the intensity ranges of the regions to be segmented. This makes it impossible to identify these regions based on the pixel intensity. In addition, the presence of intensity inhomogeneities due to the illumination variations increases the automation complexity. In this paper, we propose a method to improve the homogeneity value of the cell images leading to better segmentation results. Further, a boxcar function based Chan-Vese level set model has been proposed and implemented in a narrowband to improve the speed of segmentation.

The rest of the paper is organized as follows: Section 2 briefly describes the related work from the cell biology and image processing literature. In Section 3, we give an overview of the local intensity clustering criterion which constitutes the basis of our algorithm. The proposed method is described in Section 4. The performance of the proposed algorithm was evaluated on three real biological image datasets. Section 5 contains a performance evaluation of the method and presents the results. Finally, Section 6 concludes the paper. 


\section{RELATED WORK}

Image segmentation methods are broadly classified into two types: edge based [8] and region based [18]. Region based methods have many advantages over the edge based models such as robustness to initial conditions and boundary leakage problems in images with weak boundaries [7]. But the typical region based models tend to rely on the homogeneity of the images to be segmented as they identify each region of interest by using a certain region descriptor to guide the motion of the active contour. As it is difficult to define a region descriptor for images with intensity inhomogeneities, many methods have been proposed in the past to overcome this problem. In [10] iterative B-spline fitting for estimation of shading artifacts in fluorescence images was proposed but was limited to low noise images. Moreover, the optimal choice of threshold was found by exhaustive search. [1] proposed a probabilistic framework that enabled image registration, tissue classification, and bias correction to be combined within the same generative model. A technique for optimising the model parameters was described. The authors in [9] proposed a method that separated the intensity inhomogeneity correction field into multiplicative and additive components. The additive component was proved useful for microscopic images but much less for MR images [9]. Li et al. [7] proposed a method using the local intensity clustering criterion. A variational level set framework for segmentation and bias correction of images with intensity inhomogeneities was presented.

As the major limitation of the level set method is the slow speed of the algorithm due to its iterative nature, many methods have been proposed to improve the speed of the level set method. A narrowband level set method was proposed in [15] to segment out the cancerous tissue from the healthy tissue in brain images. A narrow band region based active contours model for noisy images was proposed in [19]to improve the speed of the level set method in noisy images. Wang et al [2]proposed a periodic monotonic speed function in the level set implementation to segment the aorta from the abdominal images. The paper proposes a narrowband method to improve the speed of the Chan-Vese level set model for cell segmentation.

\section{FRAMEWORK FOR BIAS FIELD ESTIMATION}

In this section, we provide a brief overview of the segmentation algorithm by Li et al. [7]. This framework was chosen as the base for the development of the proposed algorithm because it is the most elegant and promising method available for simultaneous bias correction and segmentation. It is based on the level set representation, which makes it easily extendable from the original formulation in 2-D to 3-D.

\subsection{Image Model}

The model used to describe the images with intensity inhomogeneity is basically a multiplicative model of the artifact as given in

$$
M=b T+n
$$

where $M$ is the measured image intensity, $T$ is the true image $b$ is the component that accounts for intensity inhomogeneity.

The component $b$ is referred to as bias field (or shading image). It is assumed to be slowly varying in the entire image domain. The true image intensities $T$ are approximately constant within each class of tissue i.e. it is piecewise constant. The additive noise $n$ is assumed to be zero mean Gaussian noise. The final goal in image segmentation is to separate the image domain $\Omega$ into $N$ disjoint regions based on the observed image $M$. However, due to intensity inho- mogeneity caused by the bias field $b$, the measured intensities are not separable by using traditional intensity based methods. Li et al. [7] proposed a method for joint segmentation and bias correction. The method uses K-means clustering algorithm by minimizing a weighted objective function. Based on the model of images with intensity inhomogeneities, a local intensity clustering criterion was defined for image intensities in the neighborhood of each point. In the level set formulation, this criterion defined energy in terms of the level set functions that represent the partition of the image domain and a bias field that accounts for the intensity inhomogeneity of the image. By minimizing this energy, the method was able to segment the image and estimate the bias field simultaneously. The present work attempts to further improve the homogeneity of the images by using GL Fractional derivative.

\subsection{Local Intensity Clustering Property}

Li et al considered a circular neighborhood with a radius $\rho$ centered at each point $y$. For a slowly varying bias field $b$, the values $b(x)$ for all $x$ in the circular neighborhood are close to $b(y)$. Then, from the image model in (1),

$$
M(x)=b(y) c_{i}+n(x)
$$

For the intensities in the neighborhood, the K-means algorithm is an iterative process to minimize the clustering criterion [17] which can be written as

$$
F_{y}=\sum_{i=1}^{N} \int\left|M(x)-m_{i}\right|^{2} u_{i}(x) d x
$$

where $m_{i}$ is the cluster center of the $i^{t h}$ cluster, $u_{i}$ is the membership function of the region $\Omega_{i}$ to be determined. A clustering criterion for classifying the intensities in the neighborhood has been defined as

$$
E_{y}=\sum_{i=1}^{N} \int K(y-x)\left|M(x)-b(y) c_{i}\right|^{2} d x
$$

where $K(y-x)$ is introduced as a non-negative window function. The energy in (4) is expressed in terms of the regions $\Omega_{1}, \ldots, \Omega_{N}$. The energy $E$ is converted to a level set formulation by representing the disjoint regions with a number of level set functions.

\section{PROPOSED METHOD}

The present paper proposes a method to make bias correction of cell images more efficient and improve the homogeneity of the cell images by using Fractional derivative. The paper also proposes a method to optimize the fractional order for a given test image. Further, the paper proposes a boxcar function based Chan Vese narrowband model to improve the speed of the level set method for cell segmentation.

\subsection{Fractional Derivatives}

In cell images, the gray level value between adjacent pixels is highly correlated with significant self similarity. Such fractal like structures are often expressed by the complex texture detail features [6]. Fractional differential is an efficient method to deal with such fractal problems. Fractional Calculus is non integer order calculus. The commonly used definitions of fractional 
derivative under Euclidean measurement are Grunwald- Letnikov and Riemann-Liouville [14].

For a one dimensional signal $s(t)$ with support domain $\Omega=[d, t]$, if $\Omega$ is divided into $n$ equal parts by interval step $h=1$, then $n=\left[\frac{t-d}{h}\right]=\frac{t-d}{h}$. Then the $\nu$ order Grumwald-Letnikov (GL) based fractional differential can be expressed by:

$$
{ }_{d}^{G} D_{t}^{v}=\lim _{h \rightarrow 0} s_{h}^{(v)}(t) h^{-v} \sum_{q=0}^{n}\left[\begin{array}{c}
-v \\
r
\end{array}\right] s(t-q+h)
$$

where

$$
\left[\begin{array}{c}
-v \\
q
\end{array}\right]=\frac{(-v)(-v+1) \ldots \ldots(-v+q-1)}{\Gamma(q+1)}
$$

and $\Gamma$ is the gamma function

For a one dimensional signal $s(t)$, it has the following expression:

$$
\begin{array}{r}
s^{v}(t)=s(t)+(-v) s(t-1)+\frac{(-v)(-v+1)}{2} s(t-2) \\
+\frac{(-v)(-v+1)(-v+2)}{6} s(t-3)+\ldots \ldots \ldots \ldots \\
+\frac{\Gamma(-v+1)}{\Gamma(n+1) \Gamma(-v+n+1) s(t-n)}
\end{array}
$$

As image is a two dimensional signal, a two dimensional mask has been obtained and fractional differential operation has been achieved by linear filtering [6]. The duration of the two dimensional digital image on $x$ and $y$ coordinates has been defined as $x \in\left[x_{1}, x_{2}\right]$ and $y \in\left[y_{1}, y_{2}\right]$ respectively.

The $x-y$ partial fractional differential of $s(x, y)$ has been defined as:

$$
\begin{aligned}
\frac{\partial^{v} s(x, y)}{\partial x^{v}} \approx s(x, y) & +(-v) s(x-1, y) \\
+ & \frac{(-v)(-v+1)}{2} s(x-2, y)+\ldots \ldots \\
\frac{\partial^{v} s(x, y)}{\partial y^{v}} \approx s(x, y) & +(-v) s(x, y-1) \\
+ & \frac{(-v)(-v+1)}{2} s(x, y-2)+\ldots \ldots
\end{aligned}
$$

4.1.1 Theoretical Analysis. The Fourier Transform of the first order differential of any quadratic integrable energy signal $s(t)$ is $D s(t) . D s(t)=(D s)(w)=(i w) s(w)=d(w) s(w)$. Similarly, from GL based definition, the Fourier Transform of fractional differential of signal $D_{G L}^{v} s(t)$ is given by

$$
\begin{array}{r}
D_{G L}^{v} s(t)=s^{v}(t) \longleftrightarrow\left(D_{G L}^{v} s\right)(w)=(i w)^{v} s(w) \\
=d_{v}(w) s(w)
\end{array}
$$

where $D_{G L}^{v}$ is $v$ order differential operator. $d_{v}(w)=(i w)^{v}$ is filter function.

The filter function of fractional differential is $d_{v}(w)=(i w)^{v}=$ $|w|^{v} e^{i \theta_{v}(w)}$. The frequency response of fractional differential is shown in Figure 1. It is clear that the frequency response of fractional differential is non-linear in nature. When $v=0$, fractional differential is an all pass filter. When $v<0$, it is a fractional inte-

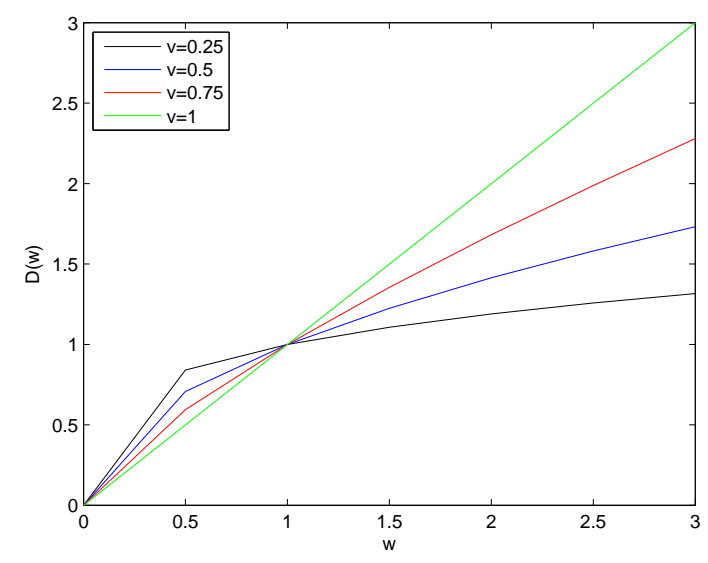

Fig. 1: Frequency Response of Fractional Differential

grator and it is low pass integral filter. When $v>0$, it is a fractional derivative operator. From the graph, it is observed that the fractional differential non linearly enhances high frequency components of a signal whereas nonlinearly inhibits low frequency components. From Fig. 1, we find that when $w>1$, the enhancement of high frequency components by fractional differential is less than integral one and the enhancement of high frequency edge components is inferior to first order one. Moreover,when $0<w<1$, the low frequency components are preserved more efficiently than the first order differential filter [6]. Hence, introducing fractional differential filter helps to extract lower frequency components in the cell images more efficiently thus improving the homogeneity value of the image.

4.1.2 Selection of Fractional Order. The fractional differential operator can be used to improve the homogeneity of the images to aid in better segmentation. But the optimal selection of the differential order is a critical problem. Based on the definition of Structural Similarity Index(SSIM), a method to find the optimal fractional order for a particular image is proposed.

Image quality assessment algorithms evaluate a test image $X$ with respect to a reference image $X_{r}$ to assess the visual similarity of the test image from the reference image. The structural similarity (SSIM) metric identifies the similarity between two images based on the assumption that the human visual system process structural features from natural images, and, therefore, the quality of an image depends on the structural similarity between the processed image and the original image.

SSIM measures visual quality with a similarity measure between two subwindows $x$ and $y$ of the two images as the product of three components: mean $m(x, y)$, variance $v(x, y)$, and cross-correlation $r(x, y)$. The two subwindows, $x$ and $y$, correspond to the same spatial window of the two images $X$ and $X_{r}$, respectively. The SSIM value for the patches $x$ and $y$ is given as

$$
\operatorname{SSIM}(x, y)=\frac{\left(2 \mu_{x} \mu_{y}+c_{1}\right)\left(2 \sigma_{x y}+c_{2}\right)}{\left(\mu_{x}^{2}+\mu_{y}^{2}+c_{1}\right)\left(\sigma_{x}^{2}+\sigma_{y}^{2}+c_{2}\right)}
$$

where $\mu_{x}$ and $\mu_{y}$ are the average of $x$ and $y$ respectively. $\sigma_{x}^{2}$ and $\sigma_{y}^{2}$ are the variance of $x$ and $y$ respectively. The overall SSIM image quality index for the images $X$ and $X_{r}$ is computed by averaging the SSIM values computed for small patches of the two images. 
The optimum fractional order for a given image is evaluated as

$$
v_{\text {opt }}=\max \left(\operatorname{SSIM}\left(I, I^{v}\right)\right)
$$

where $I$ is the original image and $I^{v}$ is the differentiated image with fractional order $v$

\subsection{Three Phase Level Set Energy Minimization for cell segmentation}

4.2.1 Chan-Vese Model with boxcar function. A level set function is a function that takes positive and negative signs that are used to represent the partition of a domain $\Omega$ into disjoint regions $\Omega_{1}, \Omega_{2}, \Omega_{N}$ depending upon the number of phases $N$ of the levels set formulation. If $\Phi$ is a level set function, then its sign defines two distinct regions.

$\Omega_{1}=[x: \Phi(x)>0]$ and $\Omega_{2}=[x: \Phi(x)<0]$ which is the partition of the domain in two different regions. As region based level set methods have many advantages over edge based methods, we have used region based method in this paper. Chan-Vese(CV) model [18] is the most commonly used region based level set model. It is based on the total squared difference of intensities of the points inside and outside of the contour. Though the CV model outperforms the traditional models, the major limitation is that it may become extremely time consuming due to periodic reinitialization of the level set.

The present work proposes a method to improve the speed of the $\mathrm{CV}$ model by introducing boxcar function in the level set equation. In the three phase level set case, the level set function $\Phi$ is used to represent the two regions $\Omega_{1}$ and $\Omega_{2}$. In this case $(N=3)$, we use two level set functions $\Phi_{1}$ and $\Phi_{2}$ to define three membership functions as:

$$
\begin{gathered}
F_{1}\left(\Phi_{1}, \Phi_{2}\right)=B\left(\Phi_{1}\right) B\left(\Phi_{2}\right) \\
F_{2}\left(\Phi_{1}, \Phi_{2}\right)=B\left(\Phi_{1}\right)\left(1-B\left(\Phi_{2}\right)\right) \\
F_{3}\left(\Phi_{1}, \Phi_{2}\right)=\left(1-B\left(\Phi_{1}\right)\right.
\end{gathered}
$$

where $B$ is Boxcar function defined as

$$
b(x)=(m-n) A f(m, n: x)=A(H(x-m)-H(x-n))
$$

where $f(m, n ; x)$ is the uniform distribution of $x$ for the interval $[m, n], \mathrm{A}$ is a constant and $H(x)$ is the heaviside step function. For the three phase case, the energy can be expressed by the following levels set formulation:

$$
E=\int \sum_{i=1}^{N}\left(\int K(y-x)\left|I(x)-b(y) c_{i}\right|^{2} d y\right) B_{i}(\Phi(x)) d x
$$

By introducing boxcar function in the evolution equation of the level set, the time taken for each computations in the evolution process of the level set is reduced as the boxcar function is unity only for a defined interval.

4.2.2 Narrowband Level Set implementation and its convergence. Throughout the evolution process of the level set, each point in the entire image has to be computed according to the evolution equation. As solving the equation for every image pixel is extremely time consuming, the level set function is calculated only on the zero level set and in the $3 \times 3$ neighbourhood of pixels. Hence, a narrowband level set algorithm has been used to further reduce the computational time for segmentation. The steps of the proposed model are as follows:

(1) Initialize the contour with a binary level set function having an arbitrary shape.

(2) Select a narrowband of pixels around the zero level set.

(3) Evolve the level set function according to (17).

(4) Continue the process until the evolution converges and there is no further change in the zero level set for several iterations.

\section{EXPERIMENTAL RESULTS AND ANALYSIS}

The proposed method was compared to the original algorithm by evaluation on real microscopy images. The results have been tested on two datasets.

\subsection{Image Datasets}

The proposed algorithm has been tested on images taken from three different databases. The first dataset has been collected from Institute of Microbial Technology (IMTECH) Chandigarh. These are the fluorescence and bright field images of THP1 cells(a human macrophage cell line). THP1 is a cell line extracted from a leukemia patient. It is used to test leukemia cell lines in analysis of proteinprotein interaction. These cells are large and round in shape. The second dataset has been obtained from the Herlev database that is publicly available. This database is provided by the Department of Pathology at Herlev University Hospital and the Department of Automation at Technical University of Denmark [5]. The third dataset is the Murphy lab database which is also publicly available [3].It consists of $2 \mathrm{D}$ images of $3 \mathrm{~T} 3$ cells.

\subsection{Results and Discussion}

In this section, we show the results for inhomogeneous cell images. These images exhibit obvious intensity inhomogeneities. We first demonstrate our method in the three phase case (i.e. $N=3$ ). The fractional differential order $\nu$ is selected based on the criteria discussed in Section 4.3. The proposed method has been implemented on the datasets discussed above.

Figure 2 shows the results of the proposed method on the brightfield image of THP1 cells. The figure also shows the histograms of the original image, bias corrected image and GL differentiated bias corrected image.

Table 1. : Comparison of homogeneity values using GLCM (Gray Level Co-occurence Matrix)

\begin{tabular}{ccc}
\hline \hline Dataset & $\begin{array}{c}\text { Homogeneity value } \\
\text { (clustering criteria) }\end{array}$ & $\begin{array}{c}\text { Homogeneity value } \\
\text { (proposed method) }\end{array}$ \\
\hline & & \\
THP1 & 0.9015 & 0.9543 \\
3T3 & 0.8667 & 0.9967 \\
\hline
\end{tabular}

The pixels of a cell image can be classified into two or three parts: cell region and background or nucleus, cytoplasm and background respectively. So, a cell image has a two peak or three peak distributed histogram model. Our method has been tested on cell images with promising results. Figure 2 shows a two peak distibuted histogram and Figure 3 shows a three peak distributed histogram 


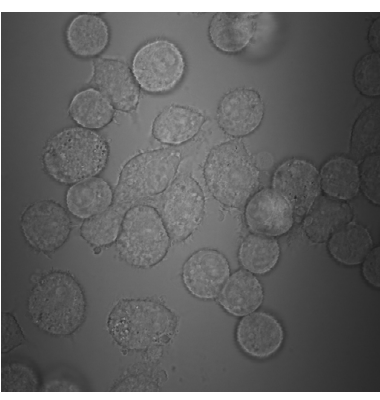

(a)

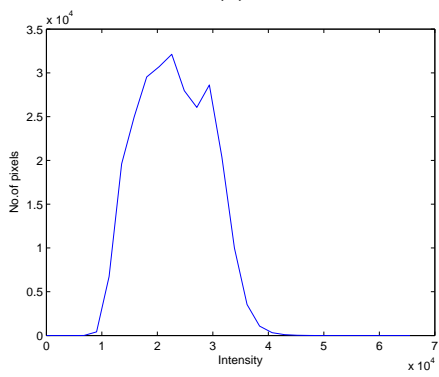

(d)

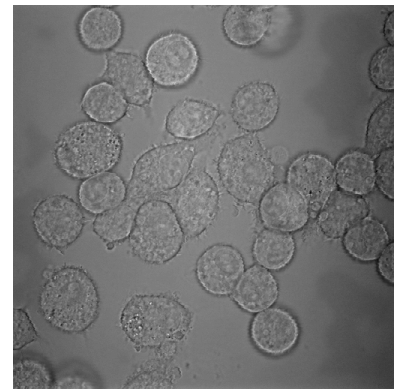

(b)

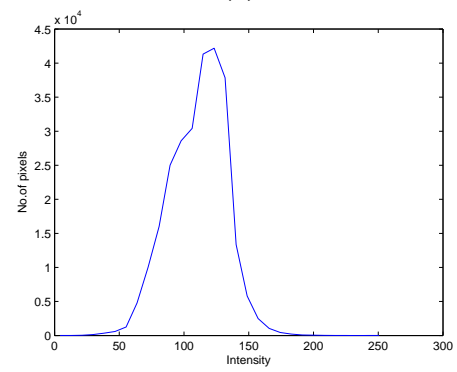

(e)

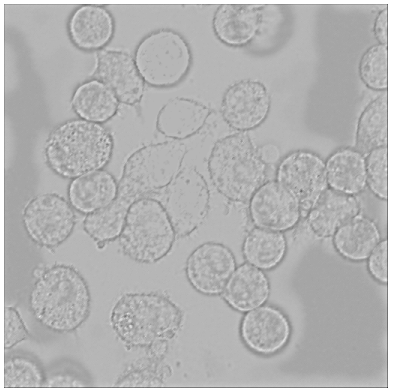

(c)

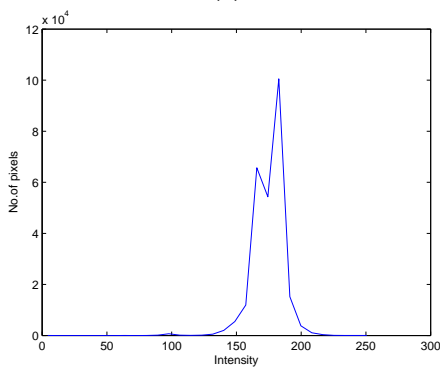

(f)

Fig. 2: Homogeneity improvement results for dataset 1 (a) Original image (b) Bias corrected image using clustering criteria (c) Bias corrected image using proposed method (d) Histogram of original image (e) Histogram of bias corrected image using clustering criteria (f) Histogram of bias corrected image using proposed method

Table 2. : Optimization of fractional order

\begin{tabular}{ccc}
\hline \hline $\operatorname{SSIM}(v=0.2)$ & $\operatorname{SSIM}(v=0.5)$ & $\operatorname{SSIM}(v=0.9)$ \\
\hline \hline 0.8238 & 0.8221 & 0.8196 \\
0.8109 & 0.8073 & 0.8078 \\
0.7754 & 0.7740 & 0.7612 \\
0.6439 & 0.6356 & 0.6302 \\
0.8348 & 0.8370 & 0.8399 \\
0.8704 & 0.8711 & 0.8712 \\
0.7611 & 0.7607 & 0.7577 \\
0.9669 & 0.9598 & 0.9581 \\
0.9699 & 0.9691 & 0.9663 \\
\hline
\end{tabular}

of a cell image. It can be seen that the GL differentiated bias corrected image shows an improvement in the homogeneity of the images. The improvement of the image quality in terms of intensity homogeneity can be shown by comparing the histograms of the bias corrected images and the GL differentiated bias corrected images. The peaks in the histograms of GL differentiated images are well defined and well separated, each corresponding to a different intensity region in the cell images. In contrast, the histograms of the bias corrected images do not have well separated peaks due to the mixture of the intensity distribution caused by bias. Moreover, the homogeneity value of the images using the GLCM statistics in Table 1 shows the improved performance of the proposed method. As the homogeneity value of the images have improved, the overlaps between the ranges of intensities of the different regions of the cells have reduced. Moreover, the fractional differential order is se- lected by (12). The SSIM values of different images for $\nu=0.2$, 0.5 and 0.9 have been tabulated in Table 2. For completeness of the paper, the optimum fractional order is also illustrated using histogram evaluation. The histograms of the test images for $\nu=0.2$ and $\nu=0.9$ have been shown in Figure 4 .

The main purpose of the above experiments was to validate the effectiveness of the proposed method for segmentation of the cells. The proposed three phase Chan Vese narrowband algorithm has been implemented to obtain the segmented images. The segmented images have been evaluated by using accuracy, precision, sensitivity and F-score [16] metrics. Table 3 and Table 4 compare the existing method and the proposed method in terms of the above mentioned metrics. The results show improved performance of the proposed method over the existing method. The computational times of the proposed method in seconds have been compared with the existing method for five different images from the databases discussed above and are tabulated in Table 5.

$$
\begin{gathered}
\text { Accuracy }=T P /(T P+F N+F P) \\
\text { Precision }=T P /(F P+T P) \\
\text { Sensitivity }=T P /(T P+F N) \\
\text { Fscore }=\frac{(\text { 2.precision.sensitivity })}{(\text { precision }+ \text { sensitivity })}
\end{gathered}
$$




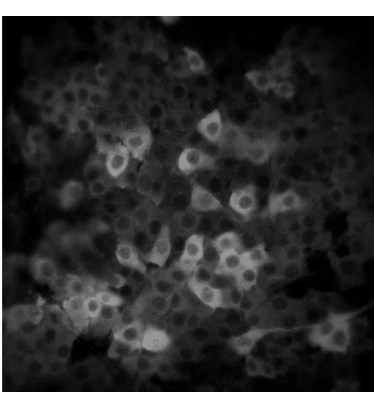

(a)

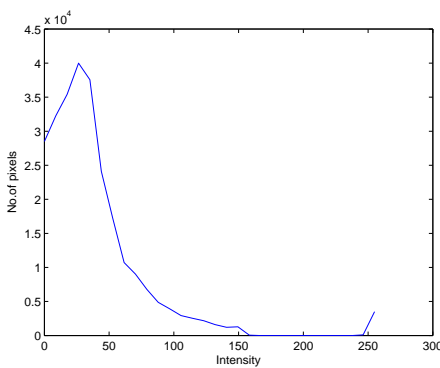

(d)

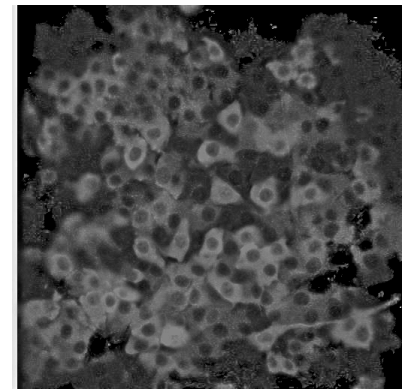

(b)

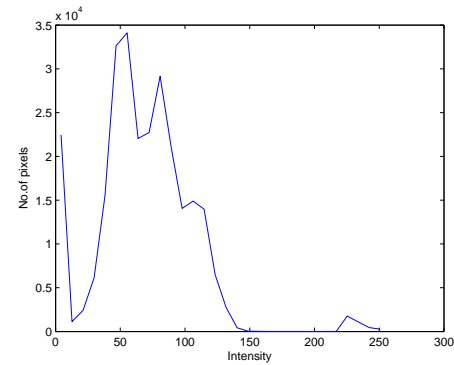

(e)

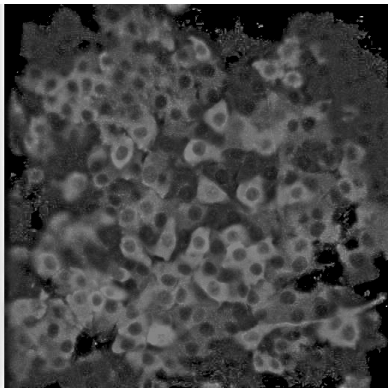

(c)

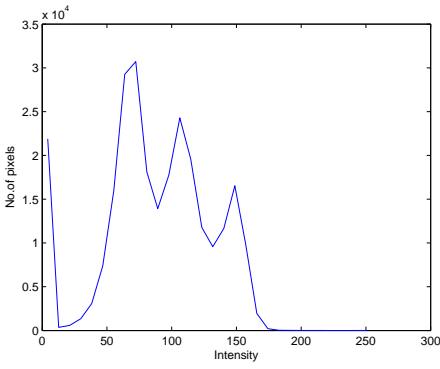

(f)

Fig. 3: Homogeneity improvement results for dataset 2 (a) Original image (b) Bias corrected image using clustering criteria (c) Bias corrected image using proposed method (d) Histogram of original image (e) Histogram of bias corrected image using clustering criteria (f) Histogram of bias corrected image using proposed method

Table 3. : Comparison of Segmentation results for dataset 1 (THP1)

\begin{tabular}{ccccc}
\hline Method & Sensitivity & Accuracy & Precision & F-score \\
\hline Existing Method & 96.28 & 95.7 & 99.4 & 82.48 \\
\hline Proposed Method & 98.57 & 98.01 & 99.4 & 98.98 \\
\hline
\end{tabular}

Table 4. : Comparison of Segmentation results for dataset 2 (3T3)

\begin{tabular}{ccccc}
\hline Method & Sensitivity & Accuracy & Precision & F-score \\
\hline Existing method & 90.29 & 80.55 & 93.54 & 89.224 \\
\hline Proposed Method & 97.14 & 89.47 & 91.89 & 94.44 \\
\hline
\end{tabular}

Table 5. : Comparison of computational times (in seconds) of the proposed method with the existing method

\begin{tabular}{cccccc}
\hline Method & THP1 & 3T3 & Columnar & Carcinoma & Severe Dysplasia \\
\hline Existing method & 12.56 & 222.09 & 535.47 & 494.57 & 535.47 \\
\hline Proposed Method & 11.40 & 209.55 & 499.45 & 401.51 & 494.24
\end{tabular}

\section{CONCLUSION}

In this paper, a fractional differential term has been introduced in the clustering criteria for improving the homogeneity of the cell images. Introducing fractional differential term in the algorithm improves the homogeneity of the image by extracting high frequency edge components and by nonlinearly preserving the low frequency components. Thus the proposed method can extract more image details as compared to the existing method. The effectiveness of the proposed method has been tested on different datsets. A method to find the optimum value of fractional order for images has been proposed. Further, a boxcar function based Chan Vese model in nar- rowband implementation has been proposed to improve the computational speed of the algorithm. The improvement in accuracy of segmentation results have been validated using accuracy, sensitivity, specificity and F-score metrics. Experimental results have demonstrated the time efficient behaviour of the proposed method as compared to the existing method.

\section{ACKNOWLEDGEMENT}

The authors would like to thank Dr. Manoj Raje, Chief Scientist (Institute of Microbial Technology, Chandigarh) for providing the cell image database.

\section{REFERENCES}

[1] Friston KJ. Ashburner J. Unified segmentation. Neuroimage., 26(3), 2005.

[2] Orjan Smedby Chunliang Wang, Hans Frimmel. Level-set based vessel segmentation accelerated with periodic monotonic speed function. SPIE Medical Imaging, 7962(2), 2011.

[3] L. P. Coelho, A. Shariff, and R. F. Murphy. Nuclear segmentation in microsope cell images: A hand-segmented dataset and comparison of algorithms. In Proceedings of the 2009 IEEE International Symposium on Biomedical Imaging, pages 518521, 2009.

[4] R. Eils and C. Athale. Computational imaging in cell biology. Cell Biology, 161(3):477-481, 2003.

[5] J. Jantzen and G. Dounias. Analysis of pap-smear image data. In Proceedings of the Nature-Inspired Smart Information Systems 2nd Annual Symposium, 2006. 


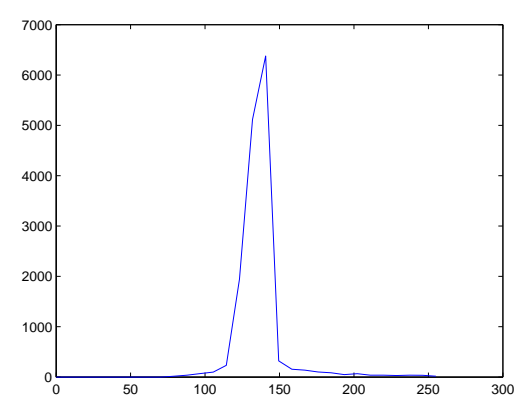

(a)

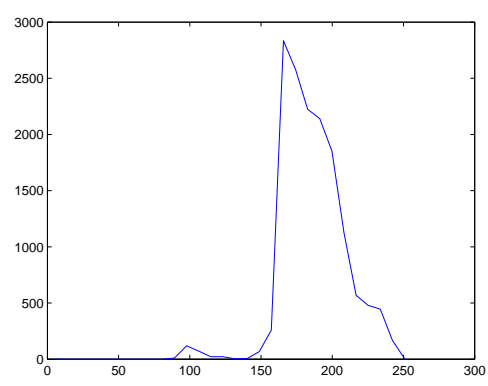

(d)

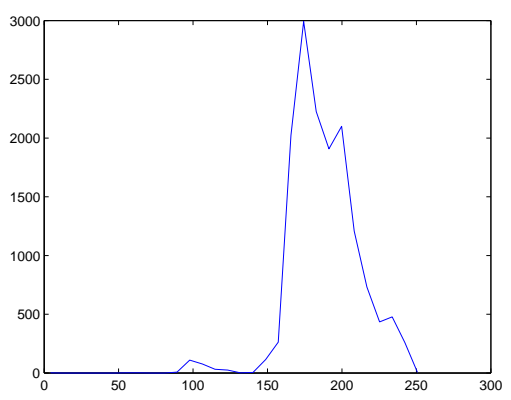

(g)

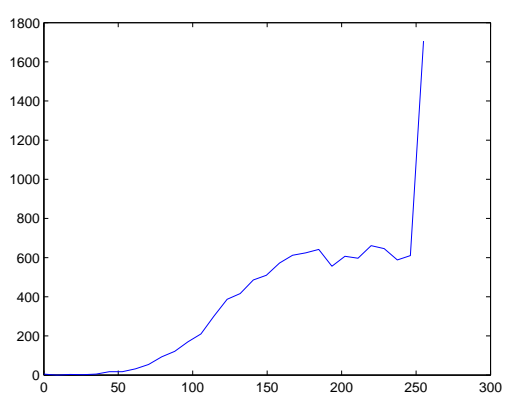

(b)

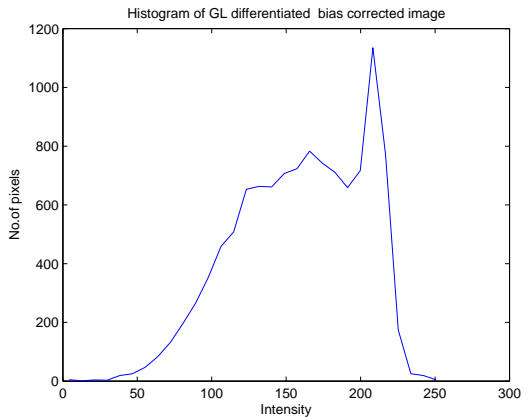

(e)

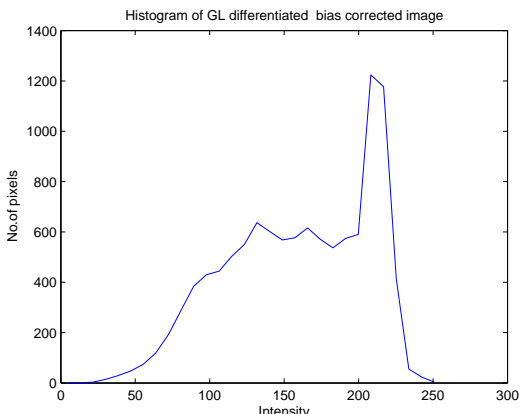

(h)

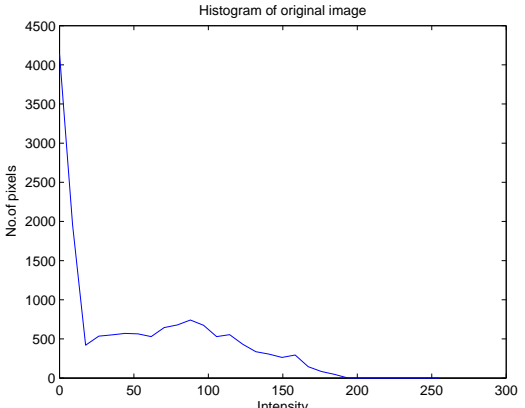

(c)

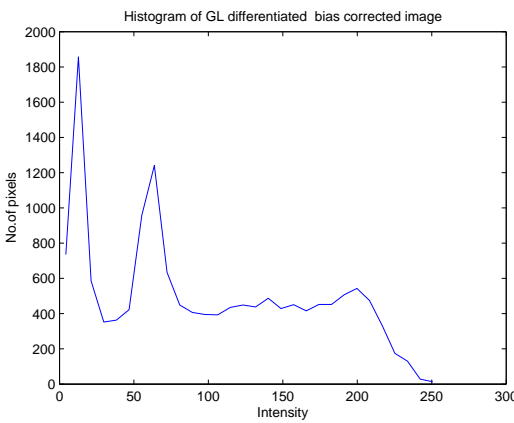

(f)

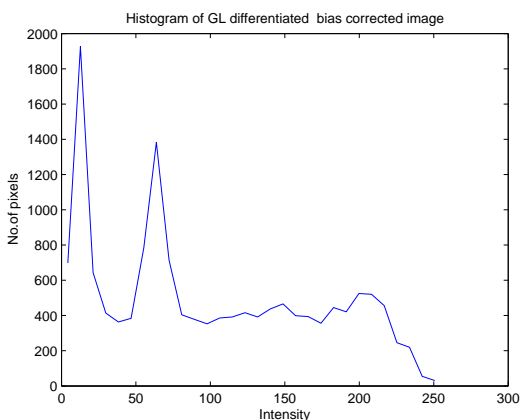

(i)

Fig. 4: Optimization of fractional order (Top row) Histograms of original images (Middle row) Histograms of bias corrected images with $\nu=0.9$ (Bottom row) Histograms of bias corrected images with $\nu=0.2$

[6] S. Kaur and J.S. Sahambi. A framework for improvement in homogeneity of fluorescence and bright field live cell images using fractional derivatives. In Computing, Communication Automation (ICCCA), 2015 International Conference on, pages 1160-1165, May 2015.

[7] Chunming Li, Rui Huang, Zhaohua Ding, J.C. Gatenby, D.N. Metaxas, and J.C. Gore. A level set method for image segmentation in the presence of intensity inhomogeneities with application to mri. Image Processing, IEEE Transactions on, 20(7):2007-2016, July 2011.

[8] Chunming Li, Chenyang Xu, Changfeng Gui, and M. D. Fox. Level set evolution without re-initialization: a new variational formulation. In Computer Vision and Pattern Recognition, 2005. CVPR 2005. IEEE Computer Society Conference on, volume 1, pages 430-436 vol. 1, June 2005.

[9] B. Likar, M.A. Viergever, and F. Pernus. Retrospective correc- tion of mr intensity inhomogeneity by information minimization. Medical Imaging, IEEE Transactions on, 20(12):13981410, Dec 2001.

[10] J. Lindblad and E. Bengtsson. A comparison of methods for estimation of intensity non uniformities in $2 \mathrm{~d}$ and $3 \mathrm{~d}$ microscope images of fluorescence stained cells. In Proceedings of the 12th Scandinavian Conference in Image Analysis(SCIA),Norway, pages 264-271, 2002.

[11] E. Meijering. Cell segmentation: 50 years down the road [life sciences]. Signal Processing Magazine, IEEE, 29(5):140145, Sept 2012.

[12] E. Meijering and O. Dzyubachyk. Tracking in cell and developmental biology. Seminars in Cell and Developmental Biology, 20(8):894-902, 2009.

[13] H. Peng. Bioimage informatics:a new area of engineering biology. Bioinformatics, 24(17):1827-1836, 2008. 
[14] Yi-Fei Pu, Ji-Liu Zhou, and Xiao Yuan. Fractional differential mask: A fractional differential-based approach for multiscale texture enhancement. Image Processing, IEEE Transactions on, 19(2):491-511, Feb 2010

[15] M. Soleimani, O. Dorn, and W.R.B. Lionheart. A narrowband level set method applied to eit in brain for cryosurgery monitoring. Biomedical Engineering, IEEE Transactions on, 53(11):2257-2264, Nov 2006.

[16] W. Tarnawski, V. Kurtcuoglu, P. Lorek, M. Bodych, J. Rotter, M. Muszkieta, L. Piwowar, D. Poulikakos, M. Majkowski, and A. Ferrari. A robust algorithm for segmenting and tracking clustered cells in time-lapse fluorescent microscopy. Biomedical and Health Informatics, IEEE Journal of, 17(4):862-869, July 2013.

[17] S. Thoedoridis and K. Koutroumbas. Pattern Recognition. Academic Press, 2003.

[18] L. Vese and T. Chan. A multiphase level set framework for image segmentation using the mumford shah model. International journal of Computer Vision, 50(3):271-293, 2002.

[19] Changming Wang Xiangfei Meng Xiaomin Xie, Aijun Zhang. Narrow band region-based active contours model for noisy color image segmentation. The Scientific World Journal, 2014(2), 2014.

[20] Xiaodong Yang, Houqiang Li, and Xiaobo Zhou. Nuclei segmentation using marker-controlled watershed, tracking using mean-shift, and kalman filter in time-lapse microscopy. Circuits and Systems I: Regular Papers, IEEE Transactions on, 53(11):2405-2414, Nov 2006. 